TRANSACTIONS OF THE

AMERICAN MATHEMATICAL SOCIETY

Volume 360, Number 8, August 2008, Pages 4425-4439

S 0002-9947(08)04456-5

Article electronically published on March 12, 2008

\title{
$L^{p}$ PROPERTIES FOR GAUSSIAN RANDOM SERIES
}

\author{
ANTOINE AYACHE AND NIKOLAY TZVETKOV
}

\begin{abstract}
Let $c=\left(c_{n}\right)_{n \in \mathbb{N}^{\star}}$ be an arbitrary sequence of $l^{2}\left(\mathbb{N}^{\star}\right)$ and let $F_{c}(\omega)$ be a random series of the type

$$
F_{c}(\omega)=\sum_{n \in \mathbb{N}^{\star}} g_{n}(\omega) c_{n} e_{n}
$$

where $\left(g_{n}\right)_{n \in \mathbb{N}^{*}}$ is a sequence of independent $\mathcal{N}_{\mathbb{C}}(0,1)$ Gaussian random variables and $\left(e_{n}\right)_{n \in \mathbb{N}^{*}}$ an orthonormal basis of $L^{2}(Y, \mathcal{M}, \mu)$ (the finite measure space $(Y, \mathcal{M}, \mu)$ being arbitrary). By using the equivalence of Gaussian moments and an integrability theorem due to Fernique, we show that a necessary and sufficient condition for $F_{c}(\omega)$ to belong to $L^{p}(Y, \mathcal{M}, \mu), p \in[2, \infty)$ for any $c \in l^{2}\left(\mathbb{N}^{\star}\right)$ almost surely is that $\sup _{n \in \mathbb{N}^{*}}\left\|e_{n}\right\|_{L^{p}(Y, \mathcal{M}, \mu)}<\infty$. One of the main motivations behind this result is the construction of a nontrivial Gibbs measure invariant under the flow of the cubic defocusing nonlinear Schrödinger equation posed on the open unit disc of $\mathbb{R}^{2}$.
\end{abstract}

\section{INTRODUCTION AND STATEMENT OF THE RESUltS}

The $d$-dimensional torus $(d \geq 1)$ is denoted by $\mathbb{T}^{d} \equiv \mathbb{R}^{d} /(2 \pi \mathbb{Z})^{d}$. Recall that the exponential functions $\exp (i n x), n \in \mathbb{Z}^{d}$, form a basis of $L^{2}\left(\mathbb{T}^{d}\right)$. Let us consider the random multivariate Fourier series

$$
F_{c}(\omega, x)=\sum_{n \in \mathbb{Z}^{d}} g_{n}(\omega) c_{n} e^{i n x},
$$

where $\left(c_{n}\right)_{n \in \mathbb{Z}^{d}}$ is a nonrandom sequence of $l^{2}\left(\mathbb{Z}^{d}\right)$ and $\left(g_{n}\right)_{n \in \mathbb{Z}^{d}}$ is a sequence of independent $\mathcal{N}_{\mathbb{C}}(0,1)$ Gaussian random variables defined on $(\Omega, \mathcal{A}, P)$, the underlying probability space. We refer to the Appendix for some clarifications concerning the series of the type (1.1). Any complex-valued centered random variable $X$ whose probability distribution function (p.d.f.) equals $\frac{1}{2 \pi \sigma^{2}} e^{-|z|^{2} / 2 \sigma^{2}}$ is called an $\mathcal{N}_{\mathbb{C}}\left(0, \sigma^{2}\right)$ Gaussian random variable 1 . It is worth noticing that, contrarily to realvalued Gaussian random variables, the variance of $X$, i.e. the quantity $\mathbb{E}\left(|X|^{2}\right)$, is not equal to $\sigma^{2}$ but $2 \sigma^{2}$. Also observe that $X$ can be written as

$$
X=X_{1}+i X_{2}
$$

where $X_{1}$ and $X_{2}$ are independent real-valued, centered Gaussian variables with variance $\sigma^{2}$. By using a classical result of Paley and Zygmund one can prove that

$$
\left\|F_{c}(\omega, \cdot)\right\|_{L^{p}\left(\mathbb{T}^{d}\right)}<\infty
$$

Received by the editors October 3, 2006.

2000 Mathematics Subject Classification. Primary 35Q55, 37K05, 37L50, 60G15, 60G50.

Key words and phrases. Eigenfunctions, Gaussian random series.

${ }^{1}$ When $\sigma=0$, then $X \equiv 0$, and its p.d.f. does not exist. However, for convenience, we suppose that 0 is a Gaussian random variable. 
almost surely (a.s.) for any $p \in[2, \infty)$. The argument to prove (1.2) based on the Khinchin inequality uses $|\exp (i n x)|=1$. As we will show in this article, however, we only need to know that the $L^{p}\left(\mathbb{T}^{d}\right)$ norms of the functions $\exp ($ inx $x), n \in \mathbb{Z}^{d}$, are bounded uniformly in $n$. This remarkable $L^{p}$ boundedness of the $L^{2}$ normalized is a very particular property of the trigonometric system. Therefore one can wonder whether the property (1.2) remains true when $\mathbb{Z}^{d}$ is replaced by $\mathbb{N}^{\star}$, the torus $\mathbb{T}^{d}$ equipped with the canonical Lebesgue measure by a finite measure space $(Y, \mathcal{M}, \mu)$ and the exponential functions by an orthonormal basis $\left(e_{n}\right)_{n \in \mathbb{N}^{\star}}$ of the Hilbert space $L^{2}(Y, \mathcal{M}, \mu)$. It turns out that the crucial point for (1.2) to hold for every sequence $c \in l^{2}\left(\mathbb{N}^{\star}\right)$ is again the uniform $L^{p}$ boundedness of $e_{n}$. Here is the precise statement.

Theorem 1. Let us fix $p \in[2, \infty)$ and a measure space $(Y, \mathcal{M}, \mu)$ with $\mu(Y)<\infty$. Let $\left(e_{n}\right)_{n \in \mathbb{N}^{\star}}$ be an orthonormal basis of $L^{2}(Y, \mathcal{M}, \mu)$. For $c=\left(c_{n}\right) \in l^{2}\left(\mathbb{N}^{\star}\right)$, we consider the random series

$$
F_{c}(\omega)=\sum_{n \in \mathbb{N}^{*}} g_{n}(\omega) c_{n} e_{n}
$$

where $\left(g_{n}\right)_{n \in \mathbb{N}^{*}}$ is a sequence of independent $\mathcal{N}_{\mathbb{C}}(0,1)$ Gaussian random variables. Then the following two statements are equivalent:

(a) For every sequence $c \in L^{2}\left(\mathbb{N}^{\star}\right)$,

$$
\left\|F_{c}(\omega)\right\|_{L^{p}(Y, \mathcal{M}, \mu)}<\infty \text { a.s. }
$$

(b) One has

$$
\sup _{n \in \mathbb{N}^{\star}}\left\|e_{n}\right\|_{L^{p}(Y, \mathcal{M}, \mu)}<\infty .
$$

In fact, Theorem 1 is a straightforward consequence of the following two results.

Theorem 2. Under the assumptions of Theorem 1,

$$
\sum_{n \in \mathbb{N}^{\star}}\left|c_{n}\right|^{2}\left\|e_{n}\right\|_{L^{p}(Y, \mathcal{M}, \mu)}^{2}<\infty
$$

implies $\left\|F_{c}(\omega)\right\|_{L^{p}(Y, \mathcal{M}, \mu)}<\infty$ a.s.

Theorem 3. Under the assumptions of Theorem 1,

$$
\sum_{n \in \mathbb{N}^{\star}}\left|c_{n}\right|^{p}\left\|e_{n}\right\|_{L^{p}(Y, \mathcal{M}, \mu)}^{p}=\infty
$$

implies $\left\|F_{c}(\omega)\right\|_{L^{p}(Y, \mathcal{M}, \mu)}=\infty$ a.s.

Remarks. (a) Theorems 2 and 3 mainly rely on the fact that a necessary and sufficient condition for

$$
\left\|F_{c}(\omega)\right\|_{L^{p}(Y, \mathcal{M}, \mu)}<\infty \text { a.s. }
$$

to be satisfied is that

$$
\mathbb{E}\left(\left\|F_{c}(\omega)\right\|_{L^{p}(Y, \mathcal{M}, \mu)}^{p}\right)=\mathbb{E}\left(\int_{Y}\left|F_{c}(\omega)\right|^{p} d \mu\right)<\infty .
$$

It is clear that (1.6) is a sufficient condition, but the fact that it is also a necessary condition may seem surprising. This is actually a consequence of the Gaussian law of $F_{c}$ and of an integrability theorem due to Fernique (see Theorem 2.1). 
(b) Another important ingredient of the proofs of Theorems 2 and 3 is the equivalence of Gaussian moments (see Lemma 2.1). Indeed, it implies that $\mu$ almost everywhere,

$$
\mathbb{E}\left(\left|F_{c}(\omega)\right|^{p}\right)=C(p)\left[\mathbb{E}\left(\left|F_{c}(\omega)\right|^{2}\right)\right]^{p / 2},
$$

where the constant $C(p)$ only depends on $p$. Then it follows from (1.3) that

$$
\mathbb{E}\left(\left|F_{c}(\omega)\right|^{p}\right)=2^{p / 2} C(p)\left(\sum_{n \in \mathbb{N}^{\star}}\left|c_{n}\right|^{2}\left|e_{n}\right|^{2}\right)^{p / 2},
$$

which makes the quantity $\mathbb{E}\left(\left\|F_{c}(\omega)\right\|_{L^{p}(Y, \mathcal{M}, \mu)}^{p}\right)$ less difficult to handle.

(c) Theorem 2 allows the construction of a nontrivial Gibbs measure on $L^{2}\left(D^{2}\right)$, invariant under the flow of the cubic defocusing nonlinear Schrödinger equation posed on the open unit disc $D^{2}$ of $\mathbb{R}^{2}$. This was one of the main motivations behind this study. We refer to the last section of the paper for details.

Let us now make the following definition.

Definition 1.1. Let $c \in l^{2}\left(\mathbb{N}^{\star}\right)$ be fixed. The critical $\left(L^{p}(Y, \mathcal{M}, \mu),\left(e_{n}\right)_{n \in \mathbb{N}^{*}}\right)$ exponent of the sequence $c$ is defined as

$$
p_{c r}(c) \equiv \sup \left(p \geq 2:\left\|F_{c}(\omega)\right\|_{L^{p}(Y, \mathcal{M}, \mu)}<\infty \text { a.s. }\right),
$$

or equivalently (in view of Remark (a)) as

$$
p_{c r}(c) \equiv \inf \left(p \geq 2:\left\|F_{c}(\omega)\right\|_{L^{p}(Y, \mathcal{M}, \mu)}=\infty \text { a.s. }\right) .
$$

The previous theorems allow us to obtain a lower and an upper bound of $p_{c r}(c)$, but are not always sufficient to exactly determine this exponent. An interesting problem would be to find a general formula allowing us to compute $p_{c r}(c)$ for any sequence $c \in l^{2}\left(\mathbb{N}^{\star}\right)$ and any orthonormal basis $\left(e_{n}\right)_{n \in \mathbb{N}^{\star}}$ of $L^{2}(Y, \mathcal{M}, \mu)$. This problem becomes less difficult to solve when one restricts to some specific situations. One of them was in fact the starting point of this work, and now we are going to describe it.

Let $D^{d} \equiv\left\{x \in \mathbb{R}^{d}:|x|<1\right\}$ be the unit ball of $\mathbb{R}^{d}$. Recall that a radial function on $D^{d}$ is a complex-valued function whose values only depend on $r \equiv|x|$ the distance to the origin. The subspace of radial functions of the Lebesgue space $L^{p}\left(D^{d}\right), p \in$ $[1, \infty)$, can be identified with the space $L^{p}\left([0,1], \mathcal{B}([0,1]), r^{d-1} d r\right)$ where $\mathcal{B}([0,1])$ is the $\sigma$-algebra of Borel sets of $[0,1]$ and $d r$ is the usual Lebesgue measure on this interval. The eigenfunctions of the Laplace operator on $L^{2}\left([0,1], \mathcal{B}([0,1]), r^{d-1} d r\right)$, denoted by $e_{n, d}, n \in \mathbb{N}^{\star}$, are closely related to Bessel functions: For any $n \in \mathbb{N}^{\star}$ and real $r>0$, one has

$$
e_{n, d}(r) \equiv \beta_{n, d}^{-1} r^{-\frac{d-2}{2}} J_{\frac{d-2}{2}}\left(z_{n, d} r\right),
$$

where $J_{\frac{d-2}{2}}$ is the Bessel function of order $\frac{d-2}{2},\left(z_{n, d}\right)_{n \in \mathbb{N}^{\star}}$ is the increasing sequence of its (simple) zeroes on $(0, \infty)$ and

$$
\beta_{n, d} \equiv\left(\int_{0}^{1}\left|J_{\frac{d-2}{2}}\left(z_{n, d} r\right)\right|^{2} r d r\right)^{\frac{1}{2}}
$$


is a normalization factor. Recall that $J_{\nu}$, the Bessel function of an arbitrary order $\nu \geq 0$, can be defined as the series

$$
J_{\nu}(r)=(r / 2)^{\nu} \sum_{n=0}^{\infty} \frac{(-1)^{n}(r / 2)^{2 n}}{n ! \Gamma(n+\nu+1)},
$$

where $\Gamma(z)=\int_{0}^{+\infty} t^{z-1} e^{-t} d t$ denotes the gamma function. It follows from the Sturm-Liouville theory that the eigenfunctions $e_{n, d}, n \in \mathbb{N}^{\star}$, form an orthonormal basis of $L^{2}\left([0,1], \mathcal{B}([0,1]), r^{d-1} d r\right)$. Thus, the analog of (1.1) (the eigenfunctions of the Laplace operator on $L^{2}\left(\mathbb{T}^{d}\right)$ being $\left.\exp (i n x), n \in \mathbb{Z}^{d}\right)$ for radial functions on $D^{d}$ is given by the random series

$$
F_{c}(\omega, r)=\sum_{n \in \mathbb{N}^{\star}} g_{n}(\omega) c_{n} e_{n, d}(r),
$$

where $\left(c_{n}\right)_{n \in \mathbb{N} *}$ is a nonrandom sequence of $l^{2}\left(\mathbb{N}^{*}\right)$ and $\left(g_{n}\right)_{n \in \mathbb{N} *}$ is again a sequence of independent $\mathcal{N}_{\mathbb{C}}(0,1)$ Gaussian random variables defined on $(\Omega, \mathcal{A}, P)$. Here is our result concerning the computation of the critical $L^{p}\left([0,1], \mathcal{B}([0,1]), r^{d-1} d r\right)$ exponent of series of the type (1.10).

Theorem 4. Let $c=\left(c_{n}\right)_{n \in N^{\star}} \in l^{2}\left(\mathbb{N}^{\star}\right)$ be a sequence satisfying the following property: There exist two constants $0<\alpha_{1} \leq \alpha_{2}$ such that for any $n \in \mathbb{N}^{\star}$,

$$
\frac{\alpha_{1}}{n} \leq c_{n} \leq \frac{\alpha_{2}}{n} .
$$

Then the critical $\left(L^{p}\left([0,1], \mathcal{B}([0,1]), r^{d-1} d r\right), e_{n, d}(r)\right)$ exponent of the corresponding series (1.10) is given by

$$
p_{c r}(c)=\frac{2 d}{d-2}
$$

Remarks. (a) If we take $c_{n}=z_{n, d}^{-1}$, then we obtain the series occurring in the Gibbs measure construction.

(b) One can also calculate $p_{c r}(c)$ for sequences for which (1.11) is replaced by

$$
\frac{c_{1}}{n^{\alpha}} \leq c_{n} \leq \frac{c_{2}}{n^{\alpha}}, \quad \alpha>\frac{1}{2} .
$$

(c) For $d \geq 3$, Theorem 4 cannot be viewed as a consequence of Theorems 2 and 3. Indeed, the latter theorems only imply that $p_{c r}(c) \in\left[\frac{2 d}{d-2}, \frac{2(d-1)}{d-3}\right]$. Actually, in order to compute the precise value of $p_{c r}(c)$, we need to use a precise description of the point-wise concentrations of $e_{n, d}$ leading to growth of the $L^{p}\left(D^{d}\right)$ norms of $e_{n, d}$. We refer to [4, 5, 6] for results giving bounds on the possible growth of the $L^{p}$ norms of $L^{2}$ normalized eigenfunctions of the Laplace operator on a compact riemannian (boundary-less) manifold.

(d) A function on $S^{d}$ (the unit sphere $\mathbb{R}^{d+1}$ ) is called zonal if its value at an arbitrary point $x$ only depends on the geodesic distance between $x$ and $x_{0}$, where $x_{0}$ is a fixed point (not depending on the function). Roughly speaking, the zonal spherical harmonics (i.e. the eigenfunctions of the Laplace-Beltrami operator on $S^{d}$ ) and the functions $e_{n, d}$ share the same properties (see [3, 7]). The latter theorem can therefore be extended to this new setting. 


\section{Proofs of the MAIN Results}

2.1. Proof of Theorem 2, The following elementary lemma is a classical result. It will be one of the main ingredients of the proof of Theorems 2 and 3 , Roughly speaking it means that the moments of a centered Gaussian random variable are equivalent.

Lemma 2.1. For any real $p>0$ there exists a constant $C(p)$ such that for any $Z$, a complex-valued centered Gaussian random variable,

$$
\mathbb{E}\left(|Z|^{p}\right)=C(p) \mathbb{E}\left(|Z|^{2}\right)^{p / 2} .
$$

As we have already noticed, the quantity

$$
\mathbb{E}\left(\left\|F_{c}\right\|_{L^{p}(Y, \mathcal{M}, \mu)}^{p}\right)=\mathbb{E}\left(\int_{Y}\left|F_{c}(\omega)\right|^{p} d \mu\right)=\int_{Y} \mathbb{E}\left(\left|F_{c}(\omega)\right|^{p}\right) d \mu,
$$

where $p \in[2, \infty)$ and $c \in l^{2}\left(\mathbb{N}^{\star}\right)$ will play a key role in the proofs of Theorems 2 and 3 . So let us first express it in a nice way.

Lemma 2.2. For any exponent $p \in[2, \infty)$ there exists a constant $d(p)$ such that for any sequence $c=\left(c_{n}\right)_{n \in \mathbb{N}^{*}}$,

$$
\mathbb{E}\left(\left\|F_{c}\right\|_{L^{p}(Y, \mathcal{M}, \mu)}^{p}\right)=d(p)\left\|\sum_{n \in \mathbb{N}^{\star}}\left|c_{n}\right|^{2}\left|e_{n}\right|^{2}\right\|_{L^{p / 2}(Y, \mathcal{M}, \mu)}^{p / 2},
$$

where $d(p)>0$ is a constant only depending on $p$.

Proof of Lemma 2.2. It follows from part (b) of Proposition 4.2 that $\mu$ almost everywhere $F_{c}(\omega)$ is an $\mathcal{N}\left(0, \sigma^{2}\right)$ Gaussian random variable, satisfying

$$
\mathbb{E}\left(\left|F_{c}(\omega)\right|^{2}\right)=2 \sigma^{2}=2 \sum_{n \in \mathbb{N}^{\star}}\left|c_{n}\right|^{2}\left|e_{n}\right|^{2} .
$$

Thus, by using Lemma 2.1] one has for any $p \in[2, \infty)$ and $\mu$ almost everywhere,

$$
\mathbb{E}\left(\left|F_{c}(\omega)\right|^{p}\right)=2^{p / 2} C(p)\left(\sum_{n \in \mathbb{N}^{*}}\left|c_{n}\right|^{2}\left|e_{n}\right|^{2}\right)^{p / 2},
$$

which implies that

$$
\begin{aligned}
\int_{Y} \mathbb{E}\left(\left|F_{c}(\omega)\right|^{p}\right) d \mu & =2^{p / 2} C(p) \int_{Y}\left(\sum_{n \in \mathbb{N}^{\star}}\left|c_{n}\right|^{2}\left|e_{n}\right|^{2}\right)^{p / 2} d \mu \\
& =2^{p / 2} C(p)\left\|\sum_{n \in \mathbb{N}^{*}}\left|c_{n}\right|^{2}\left|e_{n}\right|^{2}\right\|_{L^{p / 2}(Y, \mathcal{M}, \mu)}^{p / 2}
\end{aligned}
$$

This completes the proof of Lemma 2.2

We are now in position to prove Theorem 2, It follows from (2.2), the triangle inequality and (1.4) that

$$
\mathbb{E}\left(\left\|F_{c}\right\|_{L^{p}(Y, \mathcal{M}, \mu)}^{p}\right) \leq d(p)\left(\sum_{n \in \mathbb{N}^{\star}}\left|c_{n}\right|^{2}\left\|e_{n}\right\|_{L^{p}(Y, \mathcal{M}, \mu)}^{2}\right)^{p / 2}<\infty .
$$

Thus we get the theorem. 
2.2. Proof of Theorem 3. We will make use of the following lemma.

Lemma 2.3. For every $\alpha \geq 1$, every $N \in \mathbb{N}^{\star}$, every positive number $a_{1}, \ldots, a_{N}$, one has

$$
\left(\sum_{n=1}^{N} a_{n}\right)^{\alpha} \geq \sum_{n=1}^{N} a_{n}^{\alpha} .
$$

The following integrability theorem, due to Fernique [1, will play an important role in our analysis.

Theorem $2.1([1)$. Let $(E, \mathcal{B}(E))$ be a measurable vector space $(\mathcal{B}(E)$ denotes the $\sigma$-algebra of Borel sets of $E$ ), let $F$ be a centered Gaussian random variable with values in $E$ and let $N$ be a pseudo-norm on $E$ (the only difference between a norm and a pseudo-norm is that a pseudo-norm may take the value $\infty$ ). If the probability $P\{N(F)<\infty\}$ is strictly positive, then there exists a constant $\epsilon_{0}>0$, such that for any $0<\epsilon<\epsilon_{0}$, one has $\mathbb{E}\left[\exp \left(\epsilon N^{2}(F)\right)\right]<\infty$. In particular, for all $p<\infty$, $\mathbb{E}\left(N^{p}(F)\right)<\infty$.

Let us now give the proof of Theorem 3 Using Lemma 2.2 Lemma 2.3 and the monotone convergence theorem, we infer that

$$
\begin{aligned}
\mathbb{E}\left(\left\|F_{c}\right\|_{L^{p}(Y, \mathcal{M}, \mu)}^{p}\right) & =d(p)\left\|\sum_{n \in \mathbb{N}^{\star}}\left|c_{n}\right|^{2}\left|e_{n}\right|^{2}\right\|_{L^{p / 2}(Y, \mathcal{M}, \mu)}^{p / 2} \\
& =\lim _{N \rightarrow \infty} d(p)\left\|\sum_{n=1}^{N}\left|c_{n}\right|^{2}\left|e_{n}\right|^{2}\right\|_{L^{p / 2}(Y, \mathcal{M}, \mu)}^{p / 2} \\
& \geq d(p) \lim _{N \rightarrow \infty} \sum_{n=1}^{N}\left|c_{n}\right|^{p}\left\|e_{n}\right\|_{L^{p}(Y, \mathcal{M}, \mu)}^{p} \\
& =d(p) \sum_{n=1}^{\infty}\left|c_{n}\right|^{p}\left\|e_{n}\right\|_{L^{p}(Y, \mathcal{M}, \mu)}^{p} \\
& =\infty .
\end{aligned}
$$

Then using Theorem 2.1 with $E=L^{2}(Y, \mathcal{M}, \mu)$ and $N$ the $L^{p}(Y, \mathcal{M}, \mu)$ norm (viewed as a pseudo-norm on $L^{2}(Y, \mathcal{M}, \mu)$ ) allows us to complete the proof of Theorem 3 Indeed, if we suppose that $\left\|F_{c}\right\|_{L^{p}(Y, \mathcal{M}, \mu)}$ is not a.s. finite, then there exists a set $A$ of positive probability such that for all $\omega \in A,\left\|F_{c}(\omega)\right\|_{L^{p}(Y, \mathcal{M}, \mu)}<\infty$. Thus by the Fernique theorem $\mathbb{E}\left(\left\|F_{c}\right\|_{L^{p}(Y, \mathcal{M}, \mu)}^{p}\right)<\infty$, which contradicts (2.3).

2.3. Proof of Theorem 1. Theorem 1 is in fact a consequence of Theorems 2 and 3. It is clear that Theorem 2 gives the $(\mathrm{b})=>$ (a) implication of Theorem 1, Let us now show that (a) implies (b). Suppose ad absurdum that (a) is satisfied and

$$
\sup _{n \in \mathbb{N}^{\star}}\left\|e_{n}\right\|_{L^{p}(Y, \mathcal{M}, \mu)}=\infty .
$$

Then it follows from (2.4) that there exists a strictly increasing subsequence $k \mapsto n_{k}$, such that for every $k \in \mathbb{N}^{\star}$,

$$
\left\|e_{n_{k}}\right\|_{L^{p}(Y, \mathcal{M}, \mu)} \geq 2^{k}
$$


Finally let $\tilde{c}$ be the sequence of $l^{2}\left(\mathbb{N}^{\star}\right)$ defined for every $n$, as $\tilde{c}_{n}=2^{-k}$ when $n=n_{k}$ and $\tilde{c}_{n}=0$ elsewhere. By using (2.5) one obtains

$$
\sum_{k \in \mathbb{N}^{\star}}\left|c_{k}\right|^{p}\left\|e_{n_{k}}\right\|_{L^{p}(Y, \mathcal{M}, \mu)}^{p}=\infty .
$$

Then Theorem 3 leads to a contradiction.

2.4. Useful properties of the Bessel functions $J_{\frac{d-2}{2}}$. In this section we collect several properties of the Bessel functions useful in the proof of Theorem 4. Let us first recall three important results concerning the zeroes of $J_{\frac{d-2}{2}}$ and the behavior of this function. We refer to [2] for their proofs.

- The following inequalities are satisfied for every $n \in \mathbb{N}^{\star}$ and allow us to estimate the zeroes of $J_{\frac{d-2}{2}}$ :

$$
\alpha_{1, d} n \leq z_{n, d} \leq \alpha_{2, d} n,
$$

where $0<\alpha_{1, d} \leq \alpha_{2, d}$ are two constants.

- A sharp upper bound of $\left|J_{\frac{d-2}{2}}(r)\right|$ near the origin is given by:

$$
\left|J_{\frac{d-2}{2}}(r)\right| \leq C_{1, d} r^{\frac{d-2}{2}}
$$

where $C_{1, d}$ is a constant.

- The following equality allows us to approximate $J_{\frac{d-2}{2}}(r)$ :

$$
J_{\frac{d-2}{2}}(r)=\sqrt{\frac{2}{\pi r}} \cos (r-(d-1) \pi / 4)+R_{d}(r) .
$$

The bigger $r$ is, the better the approximation is. Indeed, the remainder $R_{d}$ satisfies

$$
\left|R_{d}(r)\right| \leq C_{2, d} r^{-\frac{3}{2}}
$$

where $C_{2, d}$ is a constant.

Let us now estimate the normalization factor $\beta_{n, d}$ introduced in (1.8).

Lemma 2.4. Let $d \geq 1$. There exist two constants $0<c_{1} \leq c_{2}$ such that for every $n \in \mathbb{N}^{\star}$,

$$
c_{1} n^{-\frac{1}{2}} \leq \beta_{n, d} \leq c_{2} n^{-\frac{1}{2}}
$$

Proof of Lemma 2.4. It follows from (1.8) and the variable change $r \rightarrow z_{n, d} r$ that

$$
\beta_{n, d}^{2}=z_{n, d}^{-2} \int_{0}^{z_{n, d}}\left|J_{\frac{d-2}{2}}(r)\right|^{2} r d r .
$$

Next using (2.6), (2.7) and (2.8), we get the inequalities

$$
\beta_{n, d}^{2} \leq C n^{-2}\left(1+\int_{1}^{\alpha_{2, d} n}\left(r^{-\frac{1}{2}}\right)^{2} r d r\right) \leq C n^{-1}
$$

and thus we obtain the upper bound part of (2.9). Let us now prove that the lower bound part holds. When $n$ is big enough, (2.10) and (2.6) entail that

$$
\beta_{n, d} \geq C n^{-1}\left(\int_{1}^{\alpha_{1, d} n}\left|J_{\frac{d-2}{2}}(r)\right|^{2} r d r\right)^{1 / 2}
$$


Next using (2.8) and the triangular inequality we get

$\beta_{n, d} \geq C n^{-1}\left(\int_{1}^{\alpha_{1, d} n} \frac{\cos ^{2}(r-(d-1) \pi / 4)}{r} r d r\right)^{1 / 2}-C n^{-1}\left(\int_{1}^{\alpha_{1, d} n} r^{-3} r d r\right)^{1 / 2}$.

Finally, using the equality $\cos ^{2} a=\frac{1+\cos 2 a}{2}$ for any real $a$, we can prove that

$$
\lim _{n \rightarrow \infty} n^{-1} \int_{1}^{\alpha_{1, d} n} \cos ^{2}(r-(d-1) \pi / 4) d r=\frac{\alpha_{1, d}}{2} .
$$

Therefore, we can conclude that for any $n$ big enough, we have $\beta_{n, d} \geq C n^{-1 / 2}$ where $C$ is a constant. This completes the proof of Lemma 2.4.

We next evaluate the $L^{p}$ norms of $e_{n, d}$.

Lemma 2.5. Let $p \in[2, \infty]$. For convenience we set, for any $n \in \mathbb{N}^{\star}$,

$$
\delta(n, p, d)= \begin{cases}1, & \text { when } 2 \leq p<\frac{2 d}{d-1}, \\ (\log (2+n))^{\frac{d-1}{2 d}}, & \text { when } p=\frac{2 d}{d-1} \\ n^{-\frac{d}{p}+\frac{d-1}{2}}, & \text { when } p>\frac{2 d}{d-1} .\end{cases}
$$

Then there exists a constant $C$ such that the inequality

$$
\left\|e_{n, d}\right\|_{L^{p}\left(D^{d}\right)} \leq C \delta(n, p, d)
$$

holds for every $n \in \mathbb{N}^{\star}$.

Proof of Lemma 2.5. Let us first study the case where $p=\infty$. It follows from (1.7) that $e_{n, d}$ can be written as

$$
e_{n, d}(r)=\beta_{n, d}^{-1} z_{n, d}^{\frac{d-2}{2}} G\left(z_{n, d} r\right),
$$

where

$$
G(r) \equiv r^{-\frac{d-2}{2}} J_{\frac{d-2}{2}}(r) .
$$

Moreover, Relations (2.7) and (2.8) allow us to show that $G$ is bounded. On the other hand Lemma 2.4 and (2.6) imply that for every $n \in \mathbb{N}^{\star}$,

$$
C_{1} n^{\frac{d-1}{2}} \leq \beta_{n, d}^{-1} z_{n, d}^{\frac{d-2}{2}} \leq C_{2} n^{\frac{d-1}{2}} .
$$

Thus, we obtain the required bound for $p=\infty$. Let us now study the case where $p<\infty$. Using (1.7) and Lemma 2.4 one has that

$$
\left\|e_{n, d}\right\|_{L^{p}\left(D^{d}\right)}^{p} \leq C_{3} n^{\frac{p}{2}} \int_{0}^{1}\left|r^{-\frac{d-2}{2}} J_{\frac{d-2}{2}}\left(z_{n, d} r\right)\right|^{p} r^{d-1} d r .
$$

Next the variable change $r \rightarrow z_{n, d} r$ and (2.6) yield

$$
\left\|e_{n, d}\right\|_{L^{p}\left(D^{d}\right)}^{p} \leq C_{4} n^{\frac{p}{2}} n^{-d+p \frac{d-2}{2}} \int_{0}^{\alpha_{2, d} n}\left|r^{-\frac{d-2}{2}} J_{\frac{d-2}{2}}(r)\right|^{p} r^{d-1} d r .
$$

Thus by using (2.7) and (2.8), we get

$$
\left\|e_{n, d}\right\|_{L^{p}\left(D^{d}\right)}^{p} \leq C_{5} n^{-d+p \frac{d-1}{2}}\left(1+\int_{1}^{\alpha_{2, d} n}\left|r^{-\frac{d-2}{2}} r^{-\frac{1}{2}}\right|^{p} r^{d-1} d r\right) .
$$

Next, the inequality, for every $n \in \mathbb{N}^{\star}$,

$$
n^{-d+p \frac{d-1}{2}} \leq(\delta(n, p, d))^{p},
$$


implies that

$$
\left\|e_{n, d}\right\|_{L^{p}\left(D^{d}\right)}^{p} \leq C_{5}(\delta(n, p, d))^{p}+C_{5} n^{-d+p \frac{d-1}{2}} \int_{1}^{\alpha_{2, d} n} r^{d-1-p \frac{d-1}{2}} d r \equiv I+I I .
$$

Finally, let us upper bound the quantity $I I$. Simple computations allow us to show that: If $p>\frac{2 d}{d-1}$, then $I I \leq C n^{-d+p \frac{d-1}{2}}=C(\delta(n, p, d))^{p}$; if $p<\frac{2 d}{d-1}$, then $I I \leq C n^{-d+p \frac{d-1}{2}} n^{d-p \frac{d-1}{2}}=C(\delta(n, p, d))^{p}$; and if $p=\frac{2 d}{d-1}$, then $I I \leq C \log (2+n)=$ $C(\delta(n, p, d))^{p}$. This completes the proof of Lemma 2.5.

Before concluding this subsection let us give a lower bound of $\left|e_{n, d}\right|$ near the origin. This bound can be viewed as a measure of the concentration of $e_{n, d}$ near the origin.

Lemma 2.6. There exist two constants $C>0$ and $\gamma>0$, such that for any integer $n \geq 1$ and real $r \in[0,1]$ satisfying $r n \leq \gamma$, one has

$$
\left|e_{n, d}(r)\right| \geq C n^{\frac{d-1}{2}} .
$$

Proof of Lemma 2.6. In view of (2.11) and (2.13), it sufficient to show that there exist $C>0$ and $\gamma>0$ two constants, such that for $r z_{n, d} \leq \gamma$ one has

$$
\left|G\left(z_{n, d} r\right)\right| \geq C .
$$

It follows from (1.9) and (2.12) that $G$ can be written as

$$
G(r)=2^{-\frac{d-2}{2}} \sum_{n=0}^{\infty} \frac{(-1)^{n}(r / 2)^{2 n}}{n ! \Gamma(n+d / 2)},
$$

which implies that $G$ is continuously differentiable on $\mathbb{R}_{+}$and

$$
G(0)=\frac{1}{2^{\frac{d-2}{2}} \Gamma(d / 2)} \neq 0 .
$$

Observe that the continuity of $G^{\prime}$, the derivative of $G$, entails that

$$
\sup _{r \in[0,1]}\left|G^{\prime}(r)\right|<\infty .
$$

Finally, using (2.16), (2.17) and the equality

$$
G\left(z_{n, d} r\right)=G(0)+z_{n, d} r \int_{0}^{1} G^{\prime}\left(t z_{n, d} r\right) d t,
$$

one obtains (2.14). This completes the proof of Lemma 2.6.

Remark 2.7. Observe that it follows from Lemma 2.6 that there exists a constant $C>0$ such that for any $n \in \mathbb{N}^{\star}$, one has

$$
\left\|e_{n, d}\right\|_{L^{p}(D)} \geq C n^{-\frac{d}{p}+\frac{d-1}{2}}
$$

which implies, when $p>\frac{2 d}{d-1}$, that

$$
\lim _{n \rightarrow \infty}\left\|e_{n, d}\right\|_{L^{p}(D)}=\infty .
$$


Proof of Remark 2.7, By using Lemma 2.6 one has

$$
\begin{aligned}
\left\|e_{n, d}\right\|_{L^{p}(D)}=\left(\int_{0}^{1}\left|e_{n, d}(r)\right|{ }^{p} r^{d-1} d r\right)^{1 / p} & \geq\left(\int_{0}^{\gamma / n}\left|e_{n, d}(r)\right|^{p} r^{d-1} d r\right)^{1 / p} \\
& \geq C n^{\frac{d-1}{2}}\left(\int_{0}^{\gamma / n} r^{d-1} d r\right)^{1 / p} \\
& =C n^{-\frac{d}{p}+\frac{d-1}{2}} .
\end{aligned}
$$

This completes the proof of Remark 2.7.

2.5. Proof of Theorem 4, Before giving the proof of Theorem 4 let us observe that Theorem 1 and the considerations of the previous section show that a new phenomenon appears when we consider the random series (1.10) instead of (1.1) : There exist sequences $c=\left(c_{n}\right)_{n \in \mathbb{N}^{\star}} \in l^{2}\left(\mathbb{N}^{\star}\right)$ for which $\left\|F_{c}(\omega)\right\|_{L^{p}\left(D^{d}\right)}<\infty$ a.s. is no longer satisfied by any exponent $p \in[2, \infty)$, but only up to the critical exponent $p_{0}=\frac{2 d}{d-1}$. More precisely, the following holds true for $F_{c}$ defined by (1.10):

(a) Let $p<\frac{2 d}{d-1}$. Then for every $c=\left(c_{n}\right)_{n \in \mathbb{N}^{\star}} \in l^{2}\left(\mathbb{N}^{\star}\right)$, one has

$$
\left\|F_{c}(\omega)\right\|_{L^{p}\left(D^{d}\right)}<\infty \text { a.s. }
$$

(b) Let $p>\frac{2 d}{d-1}$. Then there exists $c \in l^{2}\left(\mathbb{N}^{\star}\right)$ such that

$$
\left\|F_{c}(\omega)\right\|_{L^{p}\left(D^{d}\right)}=\infty \text { a.s. }
$$

Theorem 4 is a consequence of Lemma 2.5. Theorem 2. Proposition 2.8 and Re$\operatorname{mark} 2.9$.

Proposition 2.8. Let $c=\left(c_{n}\right)_{n \in \mathbb{N}^{\star}}$ be an arbitrary nonzero sequence of $l^{2}\left(\mathbb{N}^{\star}\right)$ and let $\alpha^{*}(c)$ be the quantity defined as

$$
\alpha^{*}(c)=\sup \left\{\alpha \geq 0: \liminf _{N \rightarrow \infty}\left((N+1)^{-\alpha} \sum_{n=1}^{N} n^{d-1}\left|c_{n}\right|^{2}\right)>0\right\} .
$$

Then for any exponent $p \in\left(\frac{2 d}{\alpha^{*}(c)}, \infty\right)$ one has $\left\|F_{c}(\omega)\right\|_{L^{p}\left(D^{d}\right)}=\infty$ a.s.

Observe that $\alpha^{*}(c)$ can be viewed as a measure of the speed of convergence of the series $\sum_{n=1}^{\infty}\left|c_{n}\right|^{2}$ : The lower it is, the quicker is the convergence of the series. Also observe that this quantity always belongs to $[0, d-1]$. Indeed, one clearly has

$$
\liminf _{N \rightarrow \infty}\left(\sum_{n=1}^{N} n^{d-1}\left|c_{n}\right|^{2}\right) \geq \sum_{n=1}^{\infty}\left|c_{n}\right|^{2}>0 .
$$

On the other hand, for any $\alpha>d-1$, one has

$$
\liminf _{N \rightarrow \infty}\left((N+1)^{-\alpha} \sum_{n=1}^{N} n^{d-1}\left|c_{n}\right|^{2}\right)=0
$$

since

$$
(N+1)^{-\alpha}\left(\sum_{n=1}^{N} n^{d-1}\left|c_{n}\right|^{2}\right) \leq(N+1)^{-(\alpha-d+1)} \sum_{n=1}^{\infty}\left|c_{n}\right|^{2} .
$$

Remark 2.9. Let $c$ be a sequence satisfying (1.11); then one can directly check that $\alpha^{*}(c)=d-2$. 
Proof of Proposition 2.8. The proposition is clearly satisfied when $\alpha^{*}(c)=0$, so we suppose that $\alpha^{*}(c)>0$. Let $p$ be an arbitrary number of $\left(\frac{2 d}{\alpha^{*}(c)}, \infty\right)$ and let $\delta$ be a number of $\left(0, \alpha^{*}(c)\right)$ verifying

$$
p \geq \frac{2 d}{\delta}
$$

It follows from (2.18) that there exist $N_{0} \geq 2$ and a constant $C_{1}>0$ such that the inequality

$$
\sum_{n=1}^{N} n^{d-1}\left|c_{n}\right|^{2} \geq C_{1}(N+1)^{\delta}
$$

holds for every $N \geq N_{0}$. Let us set $r_{0}=\min \left(1, \frac{\gamma}{N_{0}}\right)$. Observe that any $r \in\left(0, r_{0}\right]$ satisfies $[\gamma / r] \geq N_{0}$, where $[\gamma / r]$ denotes the integer part of $\gamma / r$. Thus, putting together Lemma 2.6 and (2.20) one obtains, for any $r \in\left(0, r_{0}\right]$,

$$
\begin{aligned}
\sum_{n \in \mathbb{N} \star}\left|c_{n}\right|^{2}\left|e_{n, d}(r)\right|^{2} & \geq \sum_{n=1}^{[\gamma / r]}\left|c_{n}\right|^{2}\left|e_{n, d}(r)\right|^{2} \\
& \geq C \sum_{n=1}^{[\gamma / r]} n^{d-1}\left|c_{n}\right|^{2} \\
& \geq C^{\prime}([\gamma / r]+1)^{\delta} \\
& \geq C^{\prime \prime} r^{-\delta} .
\end{aligned}
$$

Next using (2.2), (2.21) and (2.19) one gets

$$
\begin{aligned}
\mathbb{E}\left(\left\|F_{c}\right\|_{L^{p}\left(D^{d}\right)}^{p}\right) & \geq d(p) \int_{0}^{1}\left(\sum_{n \in \mathbb{N}^{\star}}\left|c_{n}\right|^{2}\left|e_{n, d}(r)\right|^{2}\right)^{p / 2} r^{d-1} d r \\
& \geq C_{1} \int_{0}^{r_{0}} r^{d-\frac{p \delta}{2}-1} d r \\
& =\infty .
\end{aligned}
$$

Next let us fix $\epsilon>0$. Using the last estimates and the inequality $\exp \left(\epsilon a^{2}\right) \geq C_{2} a^{p}$ for any $a \in \mathbb{R}_{+}$, where $C_{2}>0$ is a constant only depending on $\epsilon$ and $p$, one obtains

$$
\mathbb{E}\left[\exp \left(\epsilon\left\|F_{c}\right\|_{L^{p}\left(D^{d}\right)}\right]=\infty .\right.
$$

Thus it follows from part (a) of Proposition 4.2 and Theorem 2.1 that

$$
\left\|F_{c}(\omega, \cdot)\right\|_{L^{p}\left(D^{d}\right)}=\infty \text { a.s. }
$$

This completes the proof of Proposition 2.8

\section{Application to an invariant measure FOR THE CUBIC DEFOCUSING NLS ON THE DISC}

Let us now describe a consequence of Theorem 2 which was one of the main motivations behind this study. Theorem 2 allows the construction of a nontrivial Gibbs measure on $L^{2}\left(D^{2}\right)$, invariant under the flow of the cubic defocusing nonlinear Schrödinger equation posed on the open unit disc of $\mathbb{R}^{2}$, namely the partial differential equation

$$
\left(i \partial_{t}+\Delta\right) u-|u|^{2} u=0,
$$


where $\partial_{t}$ is the partial derivative with respect to time, $\Delta$ is the Laplace operator, and $u(t, x)$ is a complex-valued function defined on $\mathbb{R} \times D^{2}$. Using the same method as in $[8$, one can show that it is sufficient to take the image of the measure

$$
\exp \left(-\frac{1}{2}\left\|F_{z}(\omega)\right\|_{L^{4}\left(D^{2}\right)}^{4}\right) d P(\omega),
$$

under the function $(\Omega, \mathcal{A}) \rightarrow\left(L^{4}\left(D^{2}\right), \mathcal{B}\left(L^{4}\left(D^{2}\right)\right)\right)$,

$$
\omega \longmapsto F_{z}(\omega, r) \equiv \sqrt{2} \sum_{n \geq 1} \frac{g_{n}(\omega)}{z_{n, 2}} e_{n, 2}(r) .
$$

However, in order to show that the latter measure is nontrivial we have to prove that

$$
\left\|F_{z}(\omega)\right\|_{L^{4}\left(D^{2}\right)}<\infty \text { a.s. }
$$

Observe that (3.3) cannot be obtained by simply using the Sobolev embedding,

$$
\left\|F_{z}(\omega)\right\|_{L^{4}\left(D^{2}\right)} \leq\left\|F_{z}(\omega)\right\|_{H^{1 / 2}\left(D^{2}\right)} .
$$

Indeed, it follows from (2.6) and classical properties of i.i.d. centered Gaussian random variables that

$$
\left\|F_{z}(\omega)\right\|_{H^{1 / 2}\left(D^{2}\right)}^{2} \geq C \sum_{n \in \mathbb{N}^{\star}} \frac{\left|g_{n}(\omega)\right|^{2}}{n}=\infty \text { a.s. }
$$

However, Theorem 2 yields (3.3).

\section{Appendix}

Let $(Y, \mathcal{M}, \mu)$ be a measure space and $(\Omega, \mathcal{A}, P)$ be a probability space. For any exponent $p \in[1, \infty]$ we denote by $L^{p}(Y)$ (resp. $L^{p}(\Omega)$ ) the Banach space of complex-valued $\mathcal{M}$-measurable functions $f(r)$ defined on $Y$ (resp. of complexvalued random variables $X(\omega)$ defined on $\Omega$ ) and satisfying $\int_{Y}|f|^{p} d \mu<\infty$ (resp. $\left.\mathbb{E}\left(|Y|^{p}\right)<\infty\right)$. We denote by $L^{p}(\Omega \times Y)$ the Banach space of complex-valued, $\mathcal{A} \otimes$ $\mathcal{M}$-measurable functions $Z(\omega, r)$, defined on $\Omega \times Y$ and satisfying $\mathbb{E}\left(\int_{Y}|Z|^{p} d \mu\right)<$ $\infty$. Let $\left\{e_{n}\right\}_{n \in \mathbb{N}^{\star}}$ be an orthonormal basis of the Hilbert space $L^{2}(Y)$ (we assume that such a basis exists), let $\left(c_{n}\right)_{n \in \mathbb{N}^{\star}}$ be a nonrandom sequence of $l^{2}\left(\mathbb{N}^{\star}\right)$, i.e., a sequence of complex numbers satisfying

$$
\sum_{n=1}^{\infty}\left|c_{n}\right|^{2}<\infty
$$

and let $\left(g_{n}\right)_{n \in \mathbb{N}^{*}}$ be a sequence of independent $\mathcal{N}_{\mathbb{C}}(0,1)$ Gaussian random variables on $(\Omega, \mathcal{A}, P)$. The goal of this section is to explain why the random series

$$
F(\omega, r)=\sum_{n=1}^{\infty} g_{n}(\omega) c_{n} e_{n}(r)
$$

is well defined and to clarify in which sense it converges. Some useful properties of this random series are also given.

Proposition 4.1. For any $N \in \mathbb{N}^{\star}$ let $F_{N}$ be the function $L^{2}(\Omega \times Y)$ defined as

$$
F_{N}(\omega, r)=\sum_{n=1}^{N} g_{n}(\omega) c_{n} e_{n}(r) \text {. }
$$


Then $\left(F_{N}\right)_{N \in \mathbb{N}^{*}}$ is a Cauchy sequence in $L^{2}(\Omega \times Y)$ and the series (4.2) is defined as the limit of $\left(F_{N}\right)_{N \in \mathbb{N}^{*}}$.

Proof of Proposition 4.1. By using the fact that the $g_{n}$ 's are independent $\mathcal{N}_{\mathbb{C}}(0,1)$ Gaussian random variables and the fact that $\left\|e_{n}\right\|_{L^{2}(Y)}=1$ for any $n$, one obtains that for every $M<N$,

$$
\begin{aligned}
\left\|F_{M}-F_{N}\right\|_{L^{2}(\Omega \times Y)}^{2} & =\left\|\sum_{n=M}^{N} g_{n} c_{n} e_{n}\right\|_{L^{2}(\Omega \times Y)}^{2} \\
& =\sum_{n=M}^{N}\left\|g_{n}\right\|_{L^{2}(\Omega)}^{2}\left|c_{n}\right|^{2}\left\|e_{n}\right\|_{L^{2}(Y)}^{2} \\
& =2 \sum_{n=M}^{N}\left|c_{n}\right|^{2} .
\end{aligned}
$$

Thus it follows from (4.1) that

$$
\lim _{M \rightarrow \infty, M<N}\left\|F_{M}-F_{N}\right\|_{L^{2}(\Omega \times Y)}=0 .
$$

This completes the proof of Proposition 4.1

Proposition 4.2. The following holds true:

(a) For P-almost all $\omega \in \Omega$, the function $F(\omega)$ belongs to $L^{2}(Y)$ and the $g_{n}(\omega) c_{n}$ 's are its coordinates in the basis $\left\{e_{n}\right\}_{n \in \mathbb{N}^{*}}$ (on the exceptional negligible event, we set $F(\omega, \cdot) \equiv 0)$. Moreover, the function $F:(\Omega, \mathcal{A}) \rightarrow$ $\left(L^{2}(Y), \mathcal{B}\left(L^{2}(Y)\right)\right), \omega \mapsto F(\omega)$, where $\mathcal{B}\left(L^{2}(Y)\right)$ denotes the $\sigma$-algebra of Borel sets of $L^{2}(Y)$, is a centered Gaussian random variable with values in the Hilbert space $L^{2}(Y)$, i.e., $F$ is a measurable function and for any $h \in L^{2}(Y),\langle F, h\rangle=\int_{Y} F(r) \overline{h(r)} d \mu(r)$ is a centered complex-valued Gaussian random variable.

(b) For $\mu$-almost all $r \in Y$ the function $F(\cdot, r)$ is an $\mathcal{N}_{\mathbb{C}}\left(0, \sigma^{2}(r)\right)$ random variable, with

$$
\sigma^{2}(r)=\sum_{n=1}^{\infty}\left|c_{n}\right|^{2}\left|e_{n}(r)\right|^{2}
$$

Observe that $\sigma^{2}(r)<\infty$ for $\mu$-almost all $r$, since one has

$$
2 \int_{Y}\left(\sum_{n=1}^{\infty}\left|c_{n}\right|^{2}\left|e_{n}(r)\right|^{2}\right) d \mu(r)=\int_{Y} \mathbb{E}\left(|F(\cdot, r)|^{2}\right) d \mu(r)<\infty .
$$

Proof of Proposition 4.2. Let us first prove part (a). As the function $(\omega, r) \mapsto$ $F(\omega, r)$ belongs to $L^{2}(\Omega \times Y)$, one has $\mathbb{E}\left(\int_{Y}|F(r)|^{2} d \mu(r)\right)<\infty$, and this implies that for $P$-almost all $\omega, \int_{Y}|F(\omega, r)|^{2} d \mu(r)<\infty$, thus $F(\omega) \in L^{2}(Y)$. Let us now show that for $P$-almost all $\omega$ the $g_{n}(\omega) c_{n}$ 's are the coordinates of $F(\omega, \cdot)$ in the basis $\left\{e_{n}\right\}_{n \in \mathbb{N}^{\star}}$. In fact it is sufficient to prove for $P$-almost all $\omega$ one has

$$
\left(g_{n}(\omega) c_{n}\right)_{n \in \mathbb{N}^{\star}} \in l^{2}\left(\mathbb{N}^{\star}\right) .
$$


By using (4.1) and the fact that the $g_{n}$ 's are independent $\mathcal{N}_{\mathbb{C}}(0,1)$ Gaussian random variables one has

$$
\mathbb{E}\left(\sum_{n=1}^{\infty}\left|g_{n}\right|^{2}\left|c_{n}\right|^{2}\right)=2 \sum_{n=1}^{\infty}\left|c_{n}\right|^{2}<\infty
$$

which implies that (4.5) is satisfied a.s. On the exceptional negligible event $\mathcal{E}$ where one may have $F(\omega) \notin L^{2}(Y)$ or $\left(g_{n}(\omega) c_{n}\right)_{n \in \mathbb{N}^{\star}} \notin l^{2}\left(\mathbb{N}^{\star}\right)$ we set $F(\omega) \equiv 0$.

Let us now prove that the function $F:(\Omega, \mathcal{A}) \rightarrow\left(L^{2}(Y), \mathcal{B}\left(L^{2}(Y)\right)\right), \omega \mapsto F(\omega, \cdot)$ is measurable. As $L^{2}(Y)$ is a separable Hilbert space it is sufficient to show that for any $u=\sum_{n=1}^{\infty} a_{n} e_{n} \in L^{2}(Y)$ and any real $\rho$, the set

$$
F^{-1}(B(u, \rho)) \equiv\left\{\omega \in \Omega:\|F(\omega)-u\|_{L^{2}(Y)}<\rho\right\},
$$

belongs to $\mathcal{A}$ (note that the sequence $\left(a_{n}\right)_{n \in \mathbb{N}^{\star}}$ belongs to $l^{2}\left(\mathbb{N}^{\star}\right)$ and that $B(u, \rho$ ) is the open ball of $L^{2}(Y)$ of center $u$ a radius $\left.\rho\right)$. When $0 \notin B(u, \rho)$, the set $F^{-1}(B(u, \rho))$ can be expressed as

$$
F^{-1}(B(u, \rho)) \equiv\left\{\omega \in \Omega \backslash \mathcal{E}: \sum_{n=1}^{\infty}\left|g_{n}(\omega) c_{n}-a_{n}\right|^{2}<\rho\right\},
$$

and if $0 \in B(u, \rho)$ can be expressed as

$$
F^{-1}(B(u, \rho)) \equiv \mathcal{E} \cup\left\{\omega \in \Omega: \sum_{n=1}^{\infty}\left|g_{n}(\omega) c_{n}-a_{n}\right|^{2}<\rho\right\} .
$$

By using the fact that

$$
\sum_{n=1}^{\infty}\left|g_{n}(\omega) c_{n}-a_{n}\right|^{2}=\lim _{N \rightarrow \infty} \sum_{n=1}^{N}\left|g_{n}(\omega) c_{n}-a_{n}\right|^{2}, \quad \text { a.s. }
$$

one can see that $(\Omega, \mathcal{A}) \rightarrow(\mathbb{R}, \mathcal{B}(\mathbb{R})), \omega \mapsto \sum_{n=1}^{\infty}\left|g_{n}(\omega) c_{n}-a_{n}\right|^{2}$ is a random variable. Thus (4.6) and (4.7) imply that $F^{-1}(B(u, \rho)) \in \mathcal{A}$. Let us now prove that the measurable function $F:(\Omega, \mathcal{A}) \rightarrow\left(L^{2}(Y), \mathcal{B}\left(L^{2}(Y)\right)\right), \omega \mapsto F(\omega)$ is a centered Gaussian random variable with values in the Hilbert space $L^{2}(Y)$. Let

$$
h=\sum_{n=1}^{\infty} b_{n} e_{n}
$$

where $\left(b_{n}\right)_{n \in \mathbb{N}^{\star}} \in l^{2}\left(\mathbb{N}^{\star}\right)$ be an arbitrary function of $L^{2}(Y)$. By using (4.8) and the fact that the $g_{n}(\omega) c_{n}$ 's are, for $P$-almost all $\omega$, the coordinates of the function $F(\omega)$ in the basis $\left\{e_{n}\right\}_{n \in \mathbb{N}^{\star}}$, one has a.s., $\langle F, h\rangle=\sum_{n=1}^{\infty} g_{n} c_{n} \overline{b_{n}}$, and this implies that $\langle F, h\rangle$ is a centered complex-valued variable (recall that the limit of an almost surely convergent sequence of centered Gaussian random variables is a centered Gaussian random variable).

Finally, let us prove part (b) of the proposition. It follows from Proposition 4.1 that

$$
\lim _{N \rightarrow \infty} \int_{Y} \mathbb{E}\left(\left|F(r)-F_{N}(r)\right|^{2}\right) d \mu(r)=0 .
$$

There exists therefore a sequence $\left(N_{l}\right)_{l \in \mathbb{N}^{\star}}$ such that for $\mu$-almost all $r$, one has

$$
\lim _{l \rightarrow \infty} \mathbb{E}\left(F(r)-\left.F_{N_{l}}(r)\right|^{2}\right) .
$$


Thus by using the fact that $F_{N_{l}}(r)$ is an $\mathcal{N}_{\mathbb{C}}\left(0, \sigma_{N_{l}}^{2}(r)\right)$ Gaussian random variable, with $\sigma_{N_{l}}^{2}(r)=\sum_{n=1}^{N_{l}}\left|c_{n}\right|^{2}\left|e_{n}(r)\right|^{2}$ one obtains part (b) of the proposition (recall that the limit, in the mean square sense, of a sequence $\left(\epsilon_{n}\right)_{n \in \mathbb{N}^{\star}}$ of $\mathcal{N}\left(0, \sigma_{n}^{2}\right)$ Gaussian random variables is an $\mathcal{N}\left(0, \sigma^{2}\right)$ Gaussian random variable with $\left.\sigma^{2}=\lim _{n \rightarrow \infty} \sigma_{n}^{2}\right)$.

\section{ACKNOWLEDGEMENT}

The authors would like to thank Sophie Grivaux, Michel Ledoux and Hervé Queffélec for their advice.

\section{REFERENCES}

[1] X. Fernique, Regularité des trajectoires des fonctions aléatoires Gausiennes, Ecole d'été St. Flour 1974. MR0413238 (54:1355)

[2] L. Schwartz, Méthodes mathématiques pour les sciences physiques, Hermann, Paris 1961. MR 0143360 (26:919)

[3] E. Stein, Introduction to Fourier analysis on Euclidean spaces, Princeton University Press 1977. MR1970295 (2004a:42001)

[4] C. Sogge, Oscillatory integrals and spherical harmonics, Duke Math J. 53 (1986) 43-65. MR 835795 (87g:42026)

[5] C. Sogge, Concerning the $L^{p}$ norms of spectral clusters for second order elliptic operators on compact manifolds, J. Funct. Anal. 77 (1988) 123-138. MR.930395 (89d:35131)

[6] C. Sogge, Fourier integrals in classical analysis, Cambridge Tracts in Mathematics, 1993. MR1205579 (94c:35178)

[7] G. Szegö, Orthogonal polynomials, Colloque. Publications, AMS 1974. MR0372517 (51:8724)

[8] N. Tzvetkov, Invariant measures for the Nonlinear Schrödinger equation on the disc, Dyn. Partial Differ. Equ. 3 (2006), no. 2, 111-160. MR.2227040

[9] G. Watson, A Treatise on the Theory of Bessel Functions, 2nd edition, Cambridge Univ. Press, Cambridge, 1944. MR0010746 (6:64a)

Laboratoire Paul Painlevé, BÂt. M2, Université Lille 1, 59655 Villeneuve d'AscQ Cedex, France

E-mail address: antoine.ayache@math.univ-lille1.fr

Laboratoire Paul Painlevé, BÂt. M2, Université Lille 1, 59655 Villeneuve d'AscQ Cedex, France

E-mail address: nikolay.tzvetkov@math.univ-lille1.fr 